\title{
Influenza del trattamento della schizofrenia con neurolettici tipici o olanzapina sui costi sanitari e sugli outcomes lavorativi
}

\section{ANALISI ECONOMICA}

Angelomarco Barioglio*, Luca Guidi**, Giorgio Mariani*

\begin{abstract}
The aim of the present study was to evaluate the global treatment cost of schizophrenia with olanzapine or typical neuroleptics, according to Local Health Care Unit point of view. This analysis was performed through naturalistic observation of a cohort of schizophrenic patients referring to Ascoli Piceno ASL Department of Mental Health during 4 years (2001-2004).
\end{abstract}

During year 2003, investigators have identified a cohort of patients who were undergoing treatment switch from typical neuroleptics to olanzapine. These patients, after the switch, are observed prospectively for the next 2 years and retrospectively for the last 2 years of treament. This method allow us to collect data about 4 years of treatment: 2 years of typical neuroleptic treatment followed by 2 years of olanzapine treatment. The present work is presenting the analysis of the first 3 years of observation.

The results of our analysis are demonstrating that olanzapine treatment, through a better patient-physician alliance and with rehabilitative activities, allow lower total medical costs for the treatment of schizophrenia than typical neuroleptics. The higher acquisition cost of olanzapine versus typical neuroleptics was compensated by less hospitalizations and territorial medical interventions.

During olanzapine treatment patients followed more rehabilitative activities $(+71,26 \%, p<0,0001)$ and increased their working activities $(+39,13 \%$, $p<0,001)$.

Keywords: cost of schizophrenia, olanzapine, typical neuroleptics

Farmeconomia e percorsi terapeutici 2005; 6 (2): 93-96

\section{INTRODUZIONE}

La letteratura internazionale e nazionale ha evidenziato che il trattamento della schizofrenia con olanzapina è in grado, rispetto ai neurolettici tipici, di influenzare positivamente il rapporto medico-paziente, favorendo l'instaurarsi di una sorta di alleanza terapeutica che porta, come esito, un migliorato profilo della qualità di vita dei pazienti, una loro miglior accettazione delle attività riabilitative, un ridotto numero di ospedalizzazioni e un miglior reinserimento sociale [1-5].

Il trattamento con olanzapina influenza quindi, con la creazione e il potenziamento dell'alleanza terapeutica, il trattamento psichiatrico nei suoi vari aspetti.

In particolare attualmente la terapia riabilitativa, nei suoi vari aspetti, è assurta ad un ruolo cardine nel trattamento dei pazienti schizofrenici.
Difatti la riabilitazione psicosociale è volta a consentire a tali pazienti di recuperare le capacità perdute, di sviluppare le proprie potenzialità e di acquisire comportamenti socialmente condivisi, in modo da muoversi in modo adeguato ed efficace nel proprio spazio sociale.

Con il nostro studio abbiamo voluto verificare questi aspetti del trattamento della schizofrenia con olanzapina confrontando, in quattro anni di osservazione, 1 ' assorbimento di risorse sanitarie e l'entità del reinserimento lavorativo di una coorte di 31 pazienti trattati prima con neurolettici tipici e poi trasferiti ad olanzapina.

Il presente articolo riporta e discute i risultati relativi ai primi due anni di trattamento con neurolettici tipici e al primo anno di trattamento con olanzapina.
* Dipartimento di salute mentale della ASL di Ascoli Piceno

** Health Outcomes Research, Eli Lilly Italia 
Tabella I

Costi reali di erogazione delle prestazioni

\section{Tabella II}

Caratteristiche del campione

\begin{tabular}{lc}
\hline & Costo per giornata e/o intervento in euro \\
\hline Accesso al Centro Diurno & 46,23 \\
Visite specialistiche & 50,80 \\
Interventi infermieristici in struttura & 8,59 \\
Interventi infermieristici domiciliari & 25,14 \\
Interventi medici domiciliari & 65,81 \\
Psicoterapia & 66,72 \\
Sessioni di riabilitazione & 39,64 \\
Ricoveri (costo del DRG 430) & $2.404,62$ \\
Giornate di ricovero & 239,13 \\
\hline
\end{tabular}

\begin{tabular}{lcc}
\hline & M & F \\
\hline Sesso & 17 & 14 \\
Diagnosi schizofrenia & 17 & 14 \\
Età media (anni) & 49,6 & 50,3 \\
\hline
\end{tabular}

\section{MATERIALI E METODI}

Lo studio prevede una osservazione naturalistica di quattro anni di una coorte di 31 pazienti affetti da schizofrenia. I primi due anni di osservazione retrospettiva riguardano il periodo di trattamento con neurolettici tipici. Questi sono poi seguiti da una rilevazione prospettica di altri due anni, durante il periodo di trattamento con olanzapina.

Il campione è stato arruolato selezionando in successione temporale tutti i pazienti che nel 2003 erano in trattamento con olanzapina, presso il DSM di Ascoli Piceno, e che avevano assunto trattamento con neurolettici tipici nei due anni precedenti.

Nello studio sono state rilevate le seguenti variabili di consumo di risorse sanitarie:

- utilizzo di farmaci;

- visite specialistiche;

- interventi infermieristici in struttura e domiciliari;

- interventi medici domiciliari;

- psicoterapia;

- riabilitazione;

- uso del servizio diurno;

- ricoveri.

Per quanto riguarda invece il reinserimento sociale abbiamo rilevato sia il numero di pazienti avviati ad attività lavorativa sia l'effettivo numero di giornate di lavoro svolte nei due periodi di osservazione.

Poiché, al momento, la disponibilità di dati riguarda due anni di trattamento con neurolettici tipici e un anno di trattamento con olanzapina, siamo dovuti ricorrere ad un fattore di correzione delle incidenze di attività sanitaria e di reinserimento in modo da rendere confrontabili i due periodi. Il fattore di correzione usato prevede la semplice divisione per due di tutti i valori delle variabili rilevate durante $\mathrm{i}$ due anni di osservazione retrospettiva nel periodo di trattamento con neurolettici tipici.

Il punto di vista dell' analisi economica è quello della struttura che eroga le prestazioni sanitarie, cioè il DSM.

Tutti costi relativi alle singole prestazioni sanitarie provengono dal recente studio HONOS 2 che ha valutato i reali costi di produzione degli interventi sanitari in psichiatria [6]. Per i costi utilizzati si veda la tabella I.

Per i costi dei farmaci abbiamo utilizzato i prezzi al pubblico delle specialità medicinali riportati sull'Informatore Farmaceutico, 2003.

\section{RISULTATI}

Le caratteristiche del campione sono riportate in tabella II.

Secondo le rilevazioni effettuate, il numero medio annuale di interventi sanitari per paziente evidenzia che nel periodo di trattamento con olanzapina i pazienti hanno richiesto, in modo statisticamente significativo, meno prestazioni infermieristiche in struttura ( 20,27 contro 11,68 ; $73,54 \%$; $\mathrm{p}=0,000)$ e domiciliari $(26,23$ contro 21,03 ; $-24,73 \% ; \mathrm{p}=0,001)$, meno ricoveri $(0,48$ contro 0,13 ; $-269,23 \% ; \mathrm{p}=0,004)$ e meno giornate di ricovero (9,56 contro 1,$9 ;-403,16 \% ; \mathrm{p}=0,002)$. Durante il periodo di trattamento con olanzapina i pazienti hanno inoltre usufruito maggiormente di attività riabilitative: 5,19 vs 18,6 pari a un più $71,26 \%$ con un valore $\mathrm{p}=0,000$ (tabella III).

Non hanno invece dimostrata significatività statistica le differenze rilevate per quanto attiene agli accessi al centro diur- 


\begin{tabular}{lcccc}
\hline & Tipici & Olanzapina & P & \% olz vs tpc \\
\hline Accesso al Centro Diurno & 0,32 & 0 & 0,080 & na \\
Visite specialistiche & 7,23 & 7,74 & 0,164 & $6,59 \%$ \\
Interventi infermieristici in struttura & 20,27 & 11,68 & 0,000 & $-73,54 \%$ \\
Interventi infermieristici domiciliari & 26,23 & 21,03 & 0,001 & $-24,73 \%$ \\
Interventi medici domiciliari & 2,73 & 3,19 & 0,126 & $14,42 \%$ \\
Psicoterapia & 0,48 & 0,81 & 0,106 & $40,74 \%$ \\
Sessioni di riabilitazione & 5,19 & 18,06 & 0,000 & $71,26 \%$ \\
Ricoveri & 0,48 & 0,13 & 0,004 & $-269,23 \%$ \\
Giornate di ricovero & 9,56 & 1,9 & 0,002 & $-403,16 \%$ \\
\hline
\end{tabular}

\begin{tabular}{lcccc}
\hline & Tipici & Olanzapina & P & \% olz vs tpc \\
\hline Sessioni di riabilitazione & 5,19 & 18,06 & 0,000 & $71,26 \%$ \\
Awviamenti al lavoro & 0,11 & 0,26 & 0,286 & $57,69 \%$ \\
Giornate di lavoro & 81,29 & 133,55 & 0,001 & $39,13 \%$ \\
\hline
\end{tabular}

\begin{tabular}{lccc}
\hline & Tipici & Olanzapina & \% olz vs tpc \\
\hline Accesso al Centro Diurno & 28,87 & 0 & na \\
Visite specialistiche & 710,8 & 761,57 & $6,67 \%$ \\
Interventi infermieristici in struttura & 337,14 & 194,18 & $-73,62 \%$ \\
Interventi infermieristici domiciliari & $1.276,69$ & $1.023,87$ & $-24,69 \%$ \\
Interventi medici domiciliari & 347,36 & 406,97 & $14,65 \%$ \\
Psicoterapia & 62,51 & 104,18 & $40,00 \%$ \\
Sessioni di riabilitazione & 398,64 & $1.386,59$ & $\mathbf{7 1 , 2 5 \%}$ \\
Giornate di ricovero & $4.428,51$ & 881,22 & $-402,54 \%$ \\
Totale & $\mathbf{7 . 5 9 0 , 5 2}$ & $\mathbf{4 . 7 5 8 , 5 8}$ & $\mathbf{- 5 9 , 5 1 \%}$ \\
\hline
\end{tabular}

\begin{tabular}{lcccc}
\hline & $\begin{array}{c}\text { Costi per trattamento } \\
\text { farmacologico }\end{array}$ & $\begin{array}{c}\text { Costi per } \\
\text { interventi sanitari }\end{array}$ & Totali & Differenza \% \\
\hline Olanzapina & $2.421,01$ & $4.758,58$ & $7.179,59$ & \\
Tipici & 130,15 & $7.590,52$ & $7.720,67$ & $7,54 \%$ \\
\hline
\end{tabular}

no, il numero di visite specialistiche e gli interventi medici domiciliari.

Invece, in merito agli avviamenti ad attività lavorative (tabella IV) è da notare che nel periodo di trattamento con olanzapina, pur non raggiungendo la significatività statica, si rileva un numero quasi doppio di avviamenti al lavoro e, con significatività statistica, un incremento del numero di giornate di lavoro coerente con l'incremento di tempo dedicato ad attività riabilitative (tabella IV).

I costi conseguenti agli interventi sanitari operati nei due periodi di trattamento sono riassunti nella tabella V.

La riduzione sia del numero sia delle giornate totali di ricovero porta ad un significativo vantaggio economico per il periodo di trattamento con olanzapina. Questo vantaggio riesce a compensare $\mathrm{i}$ maggiori costi farmacologici e di riabilitazione del trattamento con l'atipico (tabella VI).

\section{DISCUSSIONE E CONCLUSIONI}

Lo studio presentato è ancora in corso di svolgimento, dovendosi completare l'ultimo anno di osservazione prospettica relativa al trattamento con olanzapina. Tuttavia, la lunghezza del periodo di osservazione sino ad ora raggiunto (tre anni complessivamente), la completezza dei dati sin qui raccolti e la chiarezza con cui l'analisi ha permesso di delineare le caratteristiche dei due diversi approcci terapeutici, ci hanno spinto a pubblicare una prima interim del nostro lavoro.

In particolare i nostri dati hanno, fino ad oggi, avvalorato la tesi che il trattamento con

\section{Tabella III}

Numero medio di interventi sanitari in un anno per paziente

\section{Tabella IV}

Dati relativi alle medie annuali per paziente delle attività riabilitative e lavorative

\section{Tabella V}

Costi medi annuali (in euro) degli interventi sanitari per paziente

\section{Tabella VI}

Costi medi totali per interventi sanitari e trattamenti farmacologici (un anno di osservazione) 
olanzapina, se pure caratterizzato da costi di acquisizione più elevati, è in grado di compensare questo svantaggio riducendo significativamente la necessità di ricorrere agli interventi d'urgenza quali i ricoveri, gli interventi domiciliari infermieristici e medici e di conseguenza i relativi costi. Questo aspetto è già ampiamente discusso in letteratura $[1,7,8]$.

Tutto ciò, oltre a portare importanti vantaggi a livello dei costi sanitari di trattamento della schizofrenia e del flusso delle attività mediche e infermieristiche nel DSM, dimostra significativi vantaggi sul fronte dell'outcome clinico. I nostri dati infatti dimostrano che durante il trattamento con l'atipico i pazienti frequentano di più e con maggior continuità le attività di riabilitazione sociale e sono più disponibili a riprendere o mantenere una attività lavorativa.

La capacità del trattamento con olanzapina di agevolare la terapia riabilitativa e l'inserimento lavorativo dei pazienti schizofrenici appare particolarmente importante alla luce della attuale centralità di tali aspetti nel trattamento della schizofrenia.

Difatti, uno degli aspetti più problematici di tale malattia mentale è l'incapacità di articolarsi armonicamente con l'ambiente di vita; i fallimenti che ne conseguono portano ad un progressivo isolamento dei pazienti, sia a causa dell'induzione di sentimenti di bassa autostima e inadeguatezza, sia a causa dell'emarginazione da parte del proprio ambiente sociale e interpersonale.
In quest'ottica la riabilitazione ha il compito di far riacquisire ai soggetti le abilità perdute e di sviluppare le loro potenzialità, portando all'apprendimento di competenze in grado di consentire di misurarsi con successo con le richieste del proprio ambiente sociale, interpersonale e lavorativo $[9,10]$.

Di particolare importanza appare l'effetto riabilitativo degli inserimenti lavorativi.

L'acquisizione di un lavoro, anche protetto, immette il paziente in un ambito non assistenziale; in questo modo egli ha un riconoscimento del proprio operato, giocando ruoli sociali normali e sperimentando relazioni di reciprocità.

I risultati del presente studio devono comunque essere valutati alla luce del fatto che allo stato attuale il periodo di osservazione delle due fasi di trattamento è ancora asimmetrico, dovendosi completare l'ultimo anno di raccolta dati. La scelta di seguire, naturalisticamente, la stessa coorte di pazienti durante le due fasi di trattamento riduce i bias di selezione del campione e assicura nel confronto una buona sovrapponibilità delle caratteristiche cliniche e sociali dei pazienti.

In conclusione, il nostro studio conferma che il trattamento olanzapina non incrementa i costi di trattamento della schizofrenia in Italia ma anzi, favorendo il rapporto tra paziente e attività riabilitative, facilita un approccio integrato del trattamento della schizofrenia in grado di migliorare le abilità funzionali e sociali dei pazienti.

\section{BIBLIOGRAFIA}

1. Hamilton SH et al. Functional outcomes in schizophrenia: a comparison of olanzapine and haloperidol in a European sample. Int Clin Psychopharmacol, 2000, 15: 245-255;

2. Rossi I et al. Valutazione dei costi di trattamento dei disturbi psicotici con olanzapina, risperidone e neurolettici tipici in un DSM Italiano. Farmacoeconomia e Percorsi Terapeutici, 2001, 2(4): 253-258;

3. Berardi $\mathrm{D}$ et al. Costo del trattamento con olanzapina nei servizi di salute mentale. Farmacoeconomia e Percorsi Terapeutici, 2002, 3(2): 65-70;

4. Revicki DA et al. Olanzapine vs haloperidol in the treatment of schizophrenia and other related psychotic disorders: quality of life and clinical outcomes of a randomized clinical trial. Quality of Life Research, 1999, 8: 417-426;

5. Grainger d et al. Resource use and quality of life of olanzapine compared with risperidone: results from an international randomized clinical trial. Eur neuropsychopharm. 1998, 8(suppl. A): s225-s226).

6. Mapelli $\mathrm{V}$ et al. Pattern di trattamento e costi nei dipartimenti di salute mentale della regione lombardia. Il progetto di ricerca HONOS2. Epidemiologia e Psichiatria Sociale. 2002, supplemento 5

7. Almond s et al. Cost analysis of the treatment of schizophrenia in the UK, a comparison of olanzapine and haloperidol. Pharmacoeconomics, 1998, 13(5Pt2):575-588;

8. Sacristan J et al. Pharmacoeconomic assessment of olanzapine in the treatment of refractory schizophrenia based on a pilot clinical study. Clin Drug Invest 1998, 15(1):29-35

9. "Metodologia della riabilitazione psicosociale" cur. G. Ba. Franco Angeli 1997

10. W. Anthony, M. Cohen, M. Farkas, C. Gagne. "Riabilitazione psichiatrica”. CIC Edizioni Internazionali 2003 